\title{
Zeta Functions and Transfer Operators for Piecewise Monotone Transformations ${ }^{\star}$
}

\author{
V. Baladi ${ }^{1}$ and G. Keller ${ }^{2, \star \star}$ \\ ${ }^{1}$ Section de Mathématiques, Université de Genève, $\mathrm{CH}-1211$ Geneva 24, Switzerland \\ 2 Institut für Angewandte Mathematik und SFB 123, Universität Heidelberg, D-6900 Heidelberg 1, \\ Federal Republic of Germany
}

\begin{abstract}
Given a piecewise monotone transformation $T$ of the interval and a piecewise continuous complex weight function $g$ of bounded variation, we prove that the Ruelle zeta function $\zeta(z)$ of $(T, g)$ extends meromorphically to $\left\{|z|<\theta^{-1}\right\}$ (where $\theta=\lim _{n \rightarrow \infty}\left\|g \circ T^{n-1} \cdots \cdots \cdot g \circ T \cdot g\right\|_{\infty}^{1 / n}$ ) and that $z$ is a pole of $\zeta$ if and only if $z^{-1}$ is an eigenvalue of the corresponding transfer operator $\mathscr{L}$. We do not assume that $\mathscr{L}$ leaves a reference measure invariant.
\end{abstract}

\section{Introduction and Statement of Results}

Suppose $T:[0,1] \rightarrow[0,1]$ is piecewise monotone, i.e., there is a finite partition $\mathscr{Z}$ of $[0,1]$ into intervals such that $T_{\mid z}$ is strictly monotone and continuous for each $Z \in \mathscr{Z}$. For a function $f:[0,1] \rightarrow \mathbb{C}$, let

$$
\begin{aligned}
& \operatorname{var}(f)=\sup \left\{\sum_{i=1}^{n}\left|f\left(a_{i}\right)-f\left(a_{i-1}\right)\right|: n \geqq 1,0 \leqq a_{0}<\cdots<a_{n} \leqq 1\right\}, \\
& \|f\|_{B V}=\operatorname{var}(f)+\sup (|f|),
\end{aligned}
$$

and denote by $B V=\left\{f:[0,1] \rightarrow \mathbb{C}\right.$ such that $\left.\|f\|_{B V}<\infty\right\}$ the space of functions of bounded variation.

Given $g \in B V$, one can define the transfer operator

$$
\mathscr{L}: B V \rightarrow B V, \quad \mathscr{L} f(x)=\sum_{y: T(y)=x}(f \cdot g)(y)=\sum_{Z \in \mathscr{Z}}(f \cdot g) \circ T_{\mid Z}^{-1}(x)
$$

and the Ruelle zeta function

$$
\zeta(z)=\exp \left(\sum_{n=1}^{\infty} \frac{z^{n}}{n} \sum_{x=T^{n} x} g_{n}(x)\right),
$$

$\star$ Research partially supported by the Fonds National Suisse

$\star \star$ Present address: Mathematisches Institut, Universität Erlangen-Nürnberg, D-8520 Erlangen, FRG 
where $g_{n}(x)=g\left(T^{n-1} x\right) \cdot \cdots \cdot g(T x) \cdot g(x)$. Let

$$
\theta=\lim _{n \rightarrow \infty}\left(\sup \left|g_{n}\right|\right)^{1 / n}
$$

The aim of this paper is to relate the poles of $\zeta(z)$ to the isolated eigenvalues of $\mathscr{L}$. Under the above assumptions, we prove in Sect. 2.

Theorem 1. The essential spectral radius of $\mathscr{L}$ is $\leqq \theta$. For each $\Theta>\theta$, the operator $\mathscr{L}$ can be decomposed as

$$
\mathscr{L}=\sum_{i=1}^{N(\Theta)} \lambda_{i} \mathscr{P}_{i} \Lambda_{i}+\mathscr{P} \mathscr{L}
$$

where $\mathscr{P}_{i}$, for $i=1, \ldots, N(\Theta)$, and $\mathscr{P}$ are mutually orthogonal projections commuting with $\mathscr{L}$ such that $\mathscr{P}+\sum_{i=1}^{N(\Theta)} \mathscr{P}_{i}=$ Id. For each $i=1, \ldots, N(\Theta)$, we have $\left|\lambda_{i}\right|>\Theta$, $\operatorname{rank}\left(\mathscr{P}_{i}\right)<\infty$, and $\Lambda_{i}=\mathscr{P}_{i}+N_{i}$, where $N_{i}$ is nilpotent and $\mathscr{P}_{i} N_{i}=N_{i} \mathscr{P}_{i}=N_{i}$. Finally $\left\|\mathscr{P} \mathscr{L}^{m}\right\|_{B V} \leqq$ const $\cdot \Theta^{m}$.

(It may happen that $\theta=\rho(\mathscr{L})$, the spectral radius of $\mathscr{L}$. In this case $N(\Theta)=0$.)

(Except in some unnatural cases the essential spectral radius of $\mathscr{L}$ is equal to $\theta$.)

Remark. In Sect. 2 we actually prove a bit more: If $\mathscr{Z}$ is a countable partition and if $\sum_{Z \in \mathscr{Z}} \sup _{Z}|g|<\infty$, then Theorem 1 still holds. Based on estimates of Rychlik (1983), a similar result was proved by Keller (1984). The main difference between our Theorem 1 and this result is that Rychlik and Keller assume the existence of a Borel measure $m$ on $[0,1]$ with full topological support and such that

$$
\int \mathscr{L} f d m=\int f d m \text { for all } f \in B V .
$$

(This implies in particular $g \geqq 0$ ).

If, in our case, $g \geqq 0$, then $\rho(\mathscr{L})$ is an eigenvalue of $\mathscr{L}$ provided $\rho(\mathscr{L})>\theta$. (This can be proved directly as in Remark 6.8 of Keller (1986), but also follows from our Theorem 2 and Pringsheim's theorem, see e.g. Landau (1946), Sect. 17). In this case one can construct a measure $m$ as above along the lines of Proposition 6.10 of Keller (1986).

To study the zeta function, we make two additional assumptions:

$$
\begin{aligned}
& g_{\mid Z} \text { is continuous for each } Z \in \mathscr{Z}, \\
& \mathscr{Z} \text { is a generating partition for } T .
\end{aligned}
$$

In Sect. 4 we prove

Theorem 2. Assume (1.3) and (1.4).

1. $\zeta(z)$ is meromorphic and nonzero in $\left\{|z|<\theta^{-1}\right\}$.

2. Suppose $|z|<\theta^{-1}$. Then $z$ is a pole of $\zeta$ of multiplicity $k$ if and only if $z^{-1}$ is an eigenvalue of $\mathscr{L}$ of multiplicity $k$.

It follows that $\zeta(z)$ is analytic and nonzero in $\left\{|z|<\rho(\mathscr{L})^{-1}\right\}$.

Remark. An analogon to Theorem 2 for subshifts of finite type and Hölder continuous $g$ was proved by Haydn (1987) under the additional assumption 
$\inf |g|>0$, precursors of his result are due to Pollicott (1986) and Ruelle (1987). Earlier work of Ruelle also points in this direction (see in particular Ruelle (1976) and Ruelle (1978), Theorem 5.29), and in a recent manuscript Ruelle (1989) proves Haydn's result without the inf $|g|>0$ assumption. Our methods rely on Haydn's work.

For piecewise monotone interval maps satisfying (1.2), Theorem 2 was conjectured by Hofbauer and Keller (1984) who proved most of it for the special case of piecewise constant $g$. This case is also investigated by Mori (1987). If $g \equiv 1$, $\zeta(z)$ is just the classical Artin-Mazur zeta function which has been studied e.g. by Milnor and Thurston (1977, 1988), Hofbauer (1985) and Preston (1988).

A good knowledge of the eigenvalues of the transfer operator is needed, e.g. for the study of resonances in dynamical systems, see Ruelle (1987), Baladi et al. (1989) and Eckmann (1988).

In order to prove Theorem 2, it is useful for technical reasons to double all points $a_{1}, \ldots, a_{N-1}$ of the set $(0,1) \backslash \bigcup_{Z \in \mathscr{Z}} \operatorname{int}(Z)$ and all their preimages as described in Sect. 1 of Hofbauer and Keller (1984) (int $(Z)$ denotes the interior of the interval $Z$ ). We call the enlarged space $X$, keep the notation $\mathscr{Z}$ for the partition, and extend $g$ and $T$ continuously to $X . X$ is totally ordered and order complete, such that it is compact for its order topology and $\mathscr{Z}$ is now an open (and closed) partition. This will be advantageous in particular in Sect. 3 where we construct Markov extensions. Note also that $X$ contains points isolated from one side (the doubled points), but it does not contain completely isolated points, in fact, each nonempty open interval in $X$ is uncountable.

Since at most finitely many periodic orbits are affected by this procedure, the poles of the zeta function are still the same. One defines the space $B V$ of functions of bounded variation on $X$ and the transfer operator $\mathscr{L}$ acting on $B V$ like in the case of the unit interval. To see that the construction just described does not change the spectral properties of $\mathscr{L}$ outside $\{|\lambda| \leqq \theta\}$, we must check that one can identify functions $f_{1}, f_{2} \in B V$ for which $\left\{x: f_{1}(x) \neq f_{2}(x)\right\}$ is at most countable. (Write $f_{1} \approx f_{2}$.) This is the content of the following proposition, which also gives a useful result from an "applied" point of view.

Proposition 1.1. Assume (1.3) and let $f \in B V$.

1. If there exist $a k \geqq 1$ and $a|\lambda|>\theta$ such that $(\mathscr{L}-\lambda)^{k} f=0$ and $f \approx 0$, then $f(x)=0$ for all $x \in X$.

2. If there exist $a k \geqq 1$ and $a \lambda \neq 0$ such that $(\mathscr{L}-\lambda)^{k} f \approx 0$, then there is $f_{1} \in B V$ such that $f_{1} \approx f$ and $(\mathscr{L}-\lambda)^{k} f_{1}=0$.

Note that assumption (1.3) is needed in Theorem 2 only because of Proposition 1.1.

Since $T$ is a positively expansive continuous mapping and $g$ is continuous in the modified system, the pressure $P(T, \log (g))$ for positive $g$ is well defined by (see Theorem 1.9 in Walters (1976)):

$$
P(T, \log (g)):=\limsup _{n \rightarrow \infty} \frac{1}{n} \log \sum_{\eta \in \mathscr{P}_{n}} \sup _{\eta}\left(g_{n}\right)
$$


where

$$
\mathscr{Z}_{n}=\left\{Z_{0} \cap T^{-1} Z_{1} \cap \cdots \cap T^{-(n-1)} Z_{n-1}: Z_{i} \in \mathscr{Z} \text { for all } i\right\} .
$$

In Sect. 4 we complete the analogy with subshifts of finite type by

Theorem 3. Assume (1.3) and (1.4). Then $P(T, \log (|g|)) \geqq \log \rho(\mathscr{L})$ and, if $g \geqq 0$, then equality holds.

We end this section with the proof of Proposition 1.1:

\section{Proof of Proposition 1.1.}

1. We first consider the case $k=1$. As $\sum_{x, f(x) \neq 0}|f(x)| \leqq \operatorname{var}(f)<\infty$, we have:

$$
|f(x)|=\left|\lambda^{-m} \mathscr{L}^{m} f(x)\right| \leqq \sum_{T^{m} y=x}\left|\frac{g_{m}(y)}{\lambda^{m}}\right| \cdot|f(y)| \leqq \frac{\sup \left|g_{m}\right|}{|\lambda|^{m}} \cdot \operatorname{var}(f)
$$

for all $m>0$ and all $x$. Letting $m \rightarrow \infty$ we see that $f(x)=0$ for all $x$.

If $k>1$, we note $h:=(\mathscr{L}-\lambda)^{k-1} f$. Since $f \approx 0$, we have $h \approx 0$ and the calculation above implies $h=0$. Induction then yields $f=0$.

2. Let $\left\{0=b_{0}<b_{1}<\cdots<b_{M}=1\right\}=\bigcup_{Z=\left[c_{Z}, d_{Z}\right] \in \mathscr{Z}}\left\{c_{Z}, d_{Z}\right\}$ be the set of singularities and denote $S=\left\{T^{n} b_{i}: 0 \leqq i \leqq M, n \geqq 0\right\}$. For $h \in B V$, let

$$
\tilde{h}(x)= \begin{cases}\lim _{y \rightarrow x, y \neq x} h(y), & \text { if } x \text { is one-sided isolated in } X, \\ \frac{1}{2}\left(\lim _{y \uparrow x, y \neq x} h(y)+\lim _{y \downarrow x, y \neq x} h(y)\right), & \text { otherwise, }\end{cases}
$$

and observe that $\tilde{h}_{1}=\tilde{h}_{2}$ if $h_{1} \approx h_{2}$. Since $T:\left(c_{Z}, d_{Z}\right) \rightarrow\left(T c_{Z}, T d_{Z}\right)$ is a homeomorphism for each $Z \in \mathscr{Z}$ and because of $(1.3), \widetilde{\mathscr{L} h}(x)=\mathscr{L} \tilde{h}(x)$ for $x \in X \backslash S$, and as $T S \subseteq S$, also $\widetilde{\mathscr{L}^{k}} h(x)=\mathscr{L}^{k} \tilde{h}(x)$ for $x \in X \backslash S$ and $k \geqq 0$. Suppose now that $(\mathscr{L}-\lambda)^{k} f \approx 0$ for some $f \in B V$ and $k>0$. Decompose $(\mathscr{L}-\lambda)^{k}=(-\lambda)^{k}$ Id $+R$, such that $R$ is a linear combination of $\mathscr{L}, \mathscr{L}^{2}, \ldots, \mathscr{L}^{k}$. Then $f \approx-(-\lambda)^{-k} R f$, and hence $\tilde{f}=-(-\lambda)^{-k} \widetilde{R f}=-(-\lambda)^{-k} R \tilde{f}$ on $X \backslash S$.

We define a function $f_{2}$, which is already close to $f_{1}$ : For $x \in X \backslash S$, let $f_{2}(x)=\tilde{f}(x)$. As $T^{-1}(X \backslash S) \leqq X \backslash S,(\mathscr{L}-\lambda)^{k} f_{2}(x)=\left((-\lambda)^{k} \mathrm{Id}+R\right) f_{2}(x)=0$ for $x \in X \backslash S$. For $x \in S$ we proceed inductively: If there is $x \in S$ such that $T^{-1} x \cap S=\varnothing$, then we set $f_{2}(x)=-(-\lambda)^{-k} R f_{2}(x)$. (Observe that $R f_{2}(x)$ is a linear combination of the values $f_{2}(y), y \in \bigcup_{i=1}^{k} T^{-i}\{x\} \subseteq X \backslash S$.) We repeat this procedure with the set $S_{1}=S \backslash\{x\}$. This yields a sequence $S \supseteqq S_{1} \supseteqq S_{2} \supseteqq \cdots$, and if at each step we choose the point $x=T^{n} b_{i}$ such that $n$ is minimal, the finite or countable intersection $S^{\prime}:=\cap_{k} S_{k}$ contains no point $x$ with $T^{-1} x \cap S^{\prime}=\varnothing$. Suppose $S^{\prime} \neq \varnothing$. Since it is contained in a finite union of one-sided orbits, $S^{\prime}$ consists of finitely many periodic orbits. Suppose $x, T x, \ldots, T^{p-1} x, T^{p} x=x$ is such an orbit of period $p$. Define $f_{2}(x)$ as $f_{2}(x)=$ $(-1)^{p}(-\lambda)^{-k p} R^{p} f_{2}(x)$. (Observe that $R^{p}$ is a linear combination of $\mathscr{L}_{k}^{p}, \mathscr{L}^{2 p}, \ldots, \mathscr{L}^{k p}$, the value $R^{p} f_{2}(x)$ is a linear combination of values $f_{2}(y), y \in \bigcup_{i=1}^{k} T^{-i p}\{x\}$, and 
$\bigcup_{i=1}^{k} T^{-i p}\{x\} \cap S^{\prime}=\{x\}$.) We proceed in the same way for all $x \in S^{\prime}$ and if $q$ is a common multiple of all periods of points in $S^{\prime}$, then $f_{2}(x)=(-1)^{q}(-\lambda)^{-k q} R^{q} f_{2}(x)$ for all $x \in X$. and

Finally let $f_{1}=\frac{1}{q} \sum_{j=0}^{q-1}(-\lambda)^{-k j}(-R)^{j} f_{2}$. Then $f_{1}=f_{2}=\tilde{f}$ on $X \backslash S$, i.e. $f_{1} \approx \tilde{f} \approx f$,

$$
\begin{aligned}
(\mathscr{L}-\lambda)^{k} f_{1} & =\left((-\lambda)^{k} \mathrm{Id}+R\right) \frac{1}{q} \sum_{j=0}^{q-1}(-\lambda)^{-k j}(-R)^{j} f_{2} \\
& =\frac{1}{q} \sum_{j=0}^{q-1}(-\lambda)^{-k(j-1)}(-R)^{j} f_{2}-\frac{1}{q} \sum_{j=1}^{q}(-\lambda)^{-k(j-1)}(-R)^{j} f_{2} \\
& =\frac{1}{q}(-\lambda)^{k}\left(f_{2}-(-\lambda)^{-k q}(-R)^{q} f_{2}\right) \\
& =0 .
\end{aligned}
$$

\section{The Spectral Decomposition}

From now on, let $\mathscr{I}$ be a finite or countable family of intervals (which are subsets of some totally ordered, order complete space, cf. Hofbauer and Keller (1982), Rychlik (1983)). Let $X$ be the disjoint union of these intervals $I \in \mathscr{I}$. Suppose further there is a family $\mathscr{Z}$ of disjoint subintervals of $X$ (in particular, for each $Z \in \mathscr{Z}$ there is $I \in \mathscr{I}$ with $Z \subseteq I$ ). Define $Y=\bigcup Z$.

We study a transformation $\stackrel{Z \in \mathscr{Z}}{T: Y} \rightarrow X$ such that for each $Z \in \mathscr{Z}$ holds

$$
\exists I \in \mathscr{I} \text { such that } T(Z) \subseteq I \text { and }
$$

$T_{\mid z}$ is monotone on $Z$ and has the Darboux property (i.e., if $J \subseteq Z$

is an interval, then $T J$ is an interval).

For an interval $J \subseteq X$ and a function $f: X \rightarrow \mathbb{C}$, define

$$
\begin{gathered}
\operatorname{var}_{J}(f):=\sup \left\{\sum_{i=1}^{n}\left|f\left(a_{i}\right)-f\left(a_{i-1}\right)\right|: n \geqq 1, a_{0}<a_{1}<\cdots<a_{n}, a_{i} \in J\right\}, \\
\operatorname{var}(f):=\sum_{I \in \mathcal{I}} \operatorname{var}_{I}(f), \\
\|f\|_{\infty}:=\sum_{I \in \mathcal{I}} \sup _{I}|f|, \quad \text { and } \\
\|f\|_{B V}:=\operatorname{var}(f)+\|f\|_{\infty} .
\end{gathered}
$$

Let $B V:=\left\{f: X \rightarrow \mathbb{C}:\|f\|_{B V}<\infty\right\}$. One easily sees that $\left(B V,\|\cdot\|_{B V}\right)$ is a Banach space.

Fix $g: X \rightarrow \mathbb{C}$ and define $g_{n}$ and $\theta$ as in Sect. 1. We make the following assumption on $g$ :

$$
M:=\sup _{I \in \mathscr{I}}\left\{\operatorname{var}_{I}(g)+\sum_{\substack{Z \in \mathscr{P} \\ Z \leqq I}} \sup _{Z}|g|\right\}<\infty
$$


Associated with $T$ and $g$ is the transfer operator

$$
\mathscr{L}: B V \rightarrow B V, \quad f \mapsto \sum_{Z \in \mathscr{Z}}(f \cdot g) \circ T_{\mid Z}^{-1} .
$$

(We check below that $\mathscr{L}$ is well defined, see Lemma 2.2.)

For $\eta \in \mathscr{Z}_{n}$, let $T_{\eta}^{-n}:=\left(T_{\mid \eta}^{n}\right)^{-1}$. Then

$$
\mathscr{L}^{n} f=\sum_{\eta \in \mathscr{L} \mathscr{Z}_{n}}\left(f \cdot g_{n}\right) \circ T_{\eta}^{-n}
$$

By $|\mathscr{L}|$, we denote the transfer operator associated with $T$ and $|g|$. Finally, $\Theta>\theta$ denotes a real number arbitrarily close to $\theta$.

The following facts will be used in the sequel without further explanation: Let $J, K \subseteq X$ be intervals, $f_{1}, f_{2}: J \rightarrow \mathbb{C}$. Then:

$$
\begin{aligned}
& \operatorname{var}_{J}\left(f_{1} \cdot f_{2}\right) \leqq \operatorname{var}_{J}\left(f_{1}\right) \cdot \sup _{J}\left|f_{2}\right|+\operatorname{var}_{J}\left(f_{2}\right) \cdot \sup _{J}\left|f_{1}\right|, \\
& \operatorname{var}_{K}\left(f_{1} \cdot \chi_{J}\right) \leqq \operatorname{var}_{J}\left(f_{1}\right)+2 \cdot \sup _{J}\left|f_{1}\right| \quad \text { if } \quad J \subseteq K, \\
& \operatorname{var}_{J}\left(f_{1} \circ T\right)=\operatorname{var}_{T J}\left(f_{1}\right) \text { if } J \subseteq Z \text { for some } Z \in \mathscr{Z},
\end{aligned}
$$

finally, for $\eta \in \mathscr{Z}_{k}$,

$$
\mathscr{L}^{k} \chi_{\eta}(x)= \begin{cases}g_{k} \circ T_{\eta}^{-k}(x), & \text { if } x \in T^{k}(\eta), \\ 0, & \text { otherwise. }\end{cases}
$$

Lemma 2.1. Let $J$ be an interval, $J \subseteq \eta \in \mathscr{Z}_{m}$ and $f: J \rightarrow \mathbb{C}$. Then

$$
\left\|\mathscr{L}^{m}\left(f \cdot \chi_{J}\right)\right\|_{B V} \leqq\left(\operatorname{var}_{J}(f)+3 \sup _{J}|f|\right) \cdot \sup _{J}\left|g_{m}\right|+\sup _{J}|f| \cdot \operatorname{var}_{J}\left|g_{m}\right| \cdot
$$

Proof. Let $I \in \mathscr{I}$ be such that $T^{m} \eta \subseteq I$, then

$$
\begin{aligned}
\operatorname{var}\left(\mathscr{L}^{m}\left(f \cdot \chi_{J}\right)\right) & =\operatorname{var}_{I}\left(\left(f \cdot g_{m} \cdot \chi_{J}\right) \circ T_{\eta}^{-m}\right) \\
& \leqq \operatorname{var}_{J}\left(f \cdot g_{m}\right)+2 \sup _{J}\left|f \cdot g_{m}\right| \\
& \leqq\left(\operatorname{var}_{J}(f)+2 \sup _{J}|f|\right) \sup _{J}\left|g_{m}\right|+\sup _{J}|f| \cdot \operatorname{var}_{J}\left(g_{m}\right)
\end{aligned}
$$

and $\left\|\mathscr{L}^{m}\left(f \cdot \chi_{J}\right)\right\|_{\infty} \leqq \sup _{J}|f| \cdot \sup _{J}\left|g_{m}\right|$.

Lemma 2.2. $\mathscr{L}(B V) \subseteq B V$. Furthermore, $\|\mathscr{L}\|_{B V} \leqq 3 \cdot M$.

Proof. Let $f: X \rightarrow \mathbb{C}$. Then, by Lemma 2.1,

$$
\begin{aligned}
\|\mathscr{L} f\|_{B V} & \leqq \sum_{Z \in \mathscr{L}}\left\|\mathscr{L}\left(f \cdot \chi_{Z}\right)\right\|_{B V} \\
& \leqq \sum_{I \in \mathscr{I}}\left(\operatorname{var}_{I}(f)+3 \sup _{I}|f|\right) \cdot\left(\operatorname{var}_{I}(g)+\sum_{\substack{Z \in \mathscr{L} \\
Z \cong I}} \sup _{Z}|g|\right) \\
& \leqq M \cdot 3\|f\|_{B V} \quad \text { by }(2.3) .
\end{aligned}
$$

Remark. Since $\operatorname{var}(|g|) \leqq \operatorname{var}(g)$, the proof of Lemma 2.2 also show that $|\mathscr{L}|(B V) \subseteq$ $B V$. 
Lemma 2.3. For each $\Theta>\theta$, there exists a constant $C$ such that $\operatorname{var}_{\eta}\left(g_{m}\right) \leqq C \cdot \Theta^{m}$ for all $m>0$ and $\eta \in \mathscr{Z}_{m}$.

Proof. Choose $\theta<\bar{\Theta}<\Theta$ and fix $k>0$ such that $\sup \left|g_{l}\right| \leqq \bar{\Theta}^{l}$ for all $l \geqq k$. Consider $\eta \in \mathscr{Z}_{k(n+1)}$ and $\eta^{\prime} \in \mathscr{Z}_{k n}$ such that $T^{k} \eta \subseteq \eta^{\prime}$. Then

$$
\begin{aligned}
\operatorname{var}_{\eta}\left(g_{k(n+1)}\right) & \leqq \operatorname{var}_{\eta}\left(g_{k}\right) \cdot \sup _{\eta^{\prime}}\left|g_{n k}\right|+\sup _{\eta}\left|g_{k}\right| \cdot \operatorname{var}_{\eta^{\prime}}\left(g_{n k}\right) \\
& \leqq \bar{\Theta}^{n k} \cdot \operatorname{var}_{\eta}\left(g_{k}\right)+\bar{\Theta}^{k} \operatorname{var}_{\eta^{\prime}}\left(g_{n k}\right)
\end{aligned}
$$

and induction yields

$$
\operatorname{var}_{\eta}\left(g_{k(n+1)}\right) \leqq(n+1) \cdot \bar{\Theta}^{n k} \cdot \sup _{\bar{\eta} \in \mathscr{Z}_{k}} \operatorname{var}_{\bar{\eta}}\left(g_{k}\right)
$$

The same recursion for $k=1$ (and with $\bar{\Theta}^{k}$ replaced by $\sup |g|$ ) shows that $\sup _{\bar{n} \in \mathscr{T}} \operatorname{var}_{\bar{\eta}}\left(g_{k}\right)<\infty$ (observe (2.3)!). As $\bar{\Theta}<\Theta$, this proves the assertion for $m=k n$. $\bar{\eta} \in \mathscr{Z}_{k}$

One passes to arbitrary $m$ using once again the recursion for $k=1$.

A consequence of Lemmas 2.1 and 2.3 is

Corollary 2.4. For each $\Theta>\theta$, there exists a constant $C$ such that $\left\|\mathscr{L}^{m} \chi_{\eta}\right\|_{B V}$, $\operatorname{var}_{\eta}\left(g_{m}\right), \sup _{\eta}\left|g_{m}\right| \leqq C \cdot \Theta^{m}$, for all $m>0$ and $\eta \in \mathscr{Z}_{m}$.

Lemma 2.5. If, in addition to (2.3), $\operatorname{var}(g)<\infty$ and $\sum_{Z \in \mathscr{Z}} \sup _{Z}|g|<\infty$, then $\sum_{\eta \in \mathscr{Z}_{m}}\left\|\mathscr{L}^{m} \chi_{\eta}\right\|_{B V}<\infty$ for all $m>0$

Proof. Let $\eta \in \mathscr{Z}_{m}, T \eta \subseteq \eta^{\prime} \in \mathscr{Z}_{m-1}$. By Lemma 2.1,

$$
\begin{aligned}
& \left\|\mathscr{L}^{m} \chi_{\eta}\right\|_{B V}=\left\|\mathscr{L}^{m-1}\left(g \circ T_{\eta}^{-1} \cdot \chi_{T \eta}\right)\right\|_{B V} \\
& \leqq\left(\operatorname{var}_{\eta}(g)+3 \sup _{\eta}|g|\right) \cdot \sup _{\eta^{\prime}}\left|g_{m-1}\right|+\sup _{\eta}|g| \cdot \operatorname{var}_{\eta^{\prime}}\left(g_{m-1}\right) \\
& \leqq\left\|\mathscr{L}^{m-1} \chi_{\eta^{\prime}}\right\|_{B V} \cdot\left(\operatorname{var}_{\eta}(g)+3 \underset{\eta}{\sup }|g|\right) \text {. }
\end{aligned}
$$

Hence,

$$
\begin{aligned}
\sum_{\eta \in \mathscr{X}_{m}}\left\|\mathscr{L}^{m} \chi_{\eta}\right\|_{B V} & \leqq \sum_{\eta^{\prime} \in \mathscr{Z}_{m-1}}\left\|\mathscr{L}^{m-1} \chi_{\eta^{\prime}}\right\|_{B V} \sum_{Z \in \mathscr{Z}}\left(\operatorname{var}_{Z \cap T^{-1}{\eta^{\prime}}^{\prime}}(g)+3 \sup _{Z \cap T^{-1} \eta_{\eta^{\prime}}}|g|\right) \\
& \leqq\left(\operatorname{var}(g)+\sum_{Z \in \mathscr{Z}} \sup _{Z}|g|\right) \sum_{\eta^{\prime} \in \mathscr{Z}_{m-1}}\left\|\mathscr{L}^{m-1} \chi_{\eta^{\prime}}\right\|_{B V},
\end{aligned}
$$

and the lemma follows by induction.

For each $m>0$ and $\eta \in \mathscr{Z}_{m}$, fix some $x_{\eta} \in \eta$.

Lemma 2.6. For each $\Theta>\theta$, there exists a constant $C>0$ such that $\sum_{\eta \in \mathscr{X}_{m}} \| \mathscr{L}^{m} \chi_{\eta} \cdot f\left(x_{\eta}\right)-$ $\mathscr{L}^{m}\left(f \cdot \chi_{\eta}\right) \|_{B V} \leqq C \cdot \Theta^{m} \cdot \operatorname{var}(f)$ uniformly in $m>0$ and $f \in B V$. 
Proof. By Lemma 2.1,

$$
\begin{aligned}
& \sum_{\eta \in \mathscr{Z}_{m}}\left\|\mathscr{L}^{m}\left(\chi_{\eta} \cdot\left(f-f\left(x_{\eta}\right)\right)\right)\right\|_{B V} \leqq \sum_{\eta \in \mathscr{Z}_{m}}\left[\left(\operatorname{var}_{\eta}\left(f-f\left(x_{\eta}\right)\right)+\underset{\eta}{\sup }\left|f-f\left(x_{\eta}\right)\right|\right) \sup _{\eta}\left|g_{m}\right|\right. \\
& \left.+\sup _{\eta}\left|f-f\left(x_{\eta}\right)\right| \cdot \operatorname{var}_{\eta}\left(g_{m}\right)\right] \\
& \leqq \sum_{\eta \in \mathscr{O}_{m}} \operatorname{var}_{\eta}(f)\left(4 \sup _{\eta}\left|g_{m}\right|+\operatorname{var}_{\eta}\left(g_{m}\right)\right) \\
& \leqq \text { const } \cdot \Theta^{m} \cdot \operatorname{var}(f) \quad \text { by Corollary } 2.4 \text {. }
\end{aligned}
$$

In the sequel, it will be convenient to work with subsets $\mathscr{A}_{m}$ of $\mathscr{Z}_{m}$. Without specifying them now, we assume that there is an $L$ such that for all $m>0$,

$$
\sup _{I \in \mathcal{I}} \#\left\{\eta \in \mathscr{Z}_{m}: \eta \subseteq I, \eta \notin \mathscr{A}_{m}\right\} \leqq L<\infty .
$$

For $f \in B V$, define

$$
\alpha_{m}(f):=\sum_{\eta \in \mathscr{A}_{m}} f\left(x_{\eta}\right) \cdot \chi_{\eta}
$$

Proposition 2.7. $\left\|\mathscr{L}^{m} \alpha_{m}-\mathscr{L}^{m}\right\|_{B V} \leqq$ const. $\Theta^{m}$ if $\mathscr{A}_{m}$ satisfies (2.4).

Proof. This follows from Lemmas 2.6 and 2.1 , by observing that

$$
\begin{aligned}
& \sum_{I \in \mathscr{I}} \sum_{\substack{\eta \in \mathscr{Z}_{m} \backslash \mathscr{A}_{m} \\
\eta \cong I}}\left\|\mathscr{L}^{m}\left(f \cdot \chi_{\eta}\right)\right\|_{B V} \leqq \sum_{I \in \mathscr{I}} L \cdot \text { const } \cdot \Theta^{m} \cdot\left(\operatorname{var}_{I}(f)+4 \sup _{I}|f|\right) \\
& \leqq \text { const } \cdot \Theta^{m} \cdot\|f\|_{B V} .
\end{aligned}
$$

If the operators $\mathscr{L}^{m} \alpha_{m}(m>0)$ are compact, $\mathscr{L}$ has the following spectral decomposition (cf. Lemma VIII.8.2 of Dunford/Schwartz (1957))

$$
\mathscr{L}^{m}=\sum_{i=1}^{N(\Theta)} \lambda_{i}^{m} \mathscr{P}_{i} \Lambda_{i}^{m}+\mathscr{P} \mathscr{L}^{m}
$$

where $\lambda_{i}, \mathscr{P}_{i}, \Lambda_{i}=\mathscr{P}_{i}+N_{i}$ and $\mathscr{P}$ are as in Theorem 1 . Note that

$$
\operatorname{tr}\left(\mathscr{P}_{i} \Lambda_{i}^{m}\right)=\operatorname{tr}\left(\mathscr{P}_{i}\right)+\sum_{j=1}^{m}\left(\begin{array}{c}
m \\
j
\end{array}\right) \operatorname{tr}\left(N_{i}^{j}\right)=\operatorname{tr}\left(\mathscr{P}_{i}\right), \quad \text { if } \quad m \geqq 1 .
$$

The following result is an extension of Theorem 1 from the introduction:

Theorem 2.8. If $\operatorname{var}(g)<\infty$ and if $\sum_{Z \in \mathscr{Z}} \sup _{Z}|g|<\infty$, then the operators $\mathscr{L}^{n} \alpha_{m}$ with $\mathscr{A}_{m}=\mathscr{Z}_{m}$ are compact and $\mathscr{L}$ has the decomposition (2.6).

Proof. Since $\mathscr{L}^{m} \alpha_{m}(f)=\sum_{\eta \in \mathscr{Y}_{m}} f\left(x_{\eta}\right) \cdot \mathscr{L}^{m} \chi_{\eta}=\sum_{\eta \in \mathscr{Y}_{m}} F_{\eta}(f) \cdot \mathscr{L}^{m} \chi_{\eta}$, where the linear functionals $F_{\eta}: B V \rightarrow \mathbb{C}, f \mapsto \stackrel{\eta \in \mathscr{X}_{m}}{f}\left(x_{\eta}\right)$ have norm equal to 1 , it follows from Lemma 2.5 that $\mathscr{L}^{m} \alpha_{m}$ is of trace class and in particular compact. 


\section{Markov Extensions}

From now on we suppose that $\mathscr{I}=\{X\}$ and that $\mathscr{Z}$ is a finite partition of $X$ into compact intervals which satisfies assumption (1.4). Note that $X$ is compact.

In general $\mathscr{Z}$ is not a Markov partition for $T$. The estimate in Sect. 5, however, which is borrowed from Haydn (1987) makes essential use of the Markov property. Therefore, we extend $(X, T)$ to a system with a countable Markov partition, show how to deduce Theorem 2 for $(X, T)$ from the corresponding statement for the extension and finally prove Theorem 2 for the extension.

In a series of papers Hofbauer $(1979,1986)$ constructed Markov extensions $\left(X^{\prime}, \hat{T}\right)$ for piecewise monotone transformations $(X, T)$ : Let

$$
\begin{aligned}
\mathscr{D}_{0} & =\{X\}, \quad \mathscr{D}_{\eta}=\left\{T^{m} Z: Z \in \mathscr{Z}_{m}, 1 \leqq m \leqq n\right\}, \\
\mathscr{D} & =\left\{T^{m} Z: Z \in \mathscr{Z}_{m}, 1 \leqq m\right\}, \\
X^{\prime} & =\{(x, D): x \in D \in \mathscr{D}\} .
\end{aligned}
$$

$\mathscr{D}$ is an at most countable family of compact intervals. Furthermore, if we denote $\mathscr{E}_{n}=\mathscr{D}_{n} \backslash \mathscr{D}_{n-1}(n \geqq 2)$ then Theorem 9 of Hofbauer (1986) shows that $\# \mathscr{E}_{n} \leqq 2 \cdot \# \mathscr{Z}$ and thus \# $\mathscr{D}_{n} \leqq$ const $\cdot n$.

Note that for $Z \in \mathscr{Z}_{n}, Z^{\prime} \in \mathscr{Z}_{n}, Z^{\prime} \in \mathscr{Z}_{m}$, we have $Z \cap T^{-n} Z^{\prime} \in \mathscr{Z}_{m+n}$ and $T^{n}$ $\left(Z \cap T^{-n} Z^{\prime}\right)=T^{n} Z \cap Z^{\prime}$. Hence, if $D=T^{n} Z \in \mathscr{D}$ and if $D \cap Z^{\prime} \neq \varnothing, Z^{\prime} \in \mathscr{Z}_{m}$, then $T^{m}\left(D \cap Z^{\prime}\right)=T^{m}\left(T^{n} Z \cap Z^{\prime}\right)=T^{m+n}\left(Z \cap T^{-n} Z^{\prime}\right) \in \mathscr{D}$, and we can define $\hat{T}: X^{\prime} \rightarrow X^{\prime}$ by

$$
\hat{T}(x, D)=\left(T x, T\left(D \cap \eta_{1}[x]\right)\right),
$$

where $\eta_{n}[x]$ denotes the element of $\mathscr{Z}_{n}$ which contains $x$. A simple inductive argument shows that for $n \geqq 1$,

$$
\hat{T}^{n}(x, D)=\left(T^{n} x, T^{n}\left(D \cap \eta_{n}[x]\right)\right) \text {. }
$$

For $\hat{x}=(x, C) \in X^{\prime}$, we denote the two projections by

$$
\hat{x}=(\pi(\hat{x}), \Delta(\hat{x})) \text {. }
$$

We have $\pi \circ \hat{T}=T \circ \pi$, and if for $(x, C),(x, D) \in X^{\prime}$ there is an $n \geqq 1$ such that $\eta_{n}[x] \cap C=\eta_{n}[x] \cap D$, then $\hat{T}^{n}(x, C)=\hat{T}^{n}(x, D)$. For $C \in \mathscr{D}$, we denote $\hat{C}:=\Delta^{-1}(C)$. The equivalent of $\mathscr{I}$ in Sect. 2 is now defined by $\hat{\mathscr{D}}=\Delta^{-1}(\mathscr{D})$. If we define $\hat{\mathscr{Z}}=\hat{\mathscr{D}} \vee \pi^{-1} \mathscr{Z}$, then $\hat{T} \hat{A} \in \hat{\mathscr{D}}$ for $\hat{A} \in \hat{\mathscr{Z}}$.

Obviously, $\hat{\mathscr{Z}}_{n}=\hat{\mathscr{D}} \vee \pi^{-1} \mathscr{Z}_{n}$. We also use the notations $\hat{\mathscr{D}}_{n}=\left\{\hat{C}: C \in \mathscr{D}_{n}\right\}$ and $\hat{\mathscr{Z}}_{n}^{k}=\left\{\hat{\eta} \in \hat{\mathscr{Z}}_{n}: \exists D \in \mathscr{D}_{k}\right.$ such that $\left.\hat{\eta} \subseteq \hat{D}\right\},(n \geqq 1)$. Finally, let $e: X \rightarrow X^{\prime}, x \mapsto(x, X)$ and observe that $e(X)=\hat{X}$ is the "basis" of $X^{\prime}$.

Note that the Markov extension described above is again of the form considered in Sect. 2 (i.e., assumptions (2.1) and (2.2) are still satisfied) and that all results of that section can be applied.

The following is Lemma 2 in Hofbauer (1985) or Theorem 8 in Hofbauer (1986):

Lemma 3.1. For a subset $A$ of $X$, we denote by $\operatorname{int}(A)$ the interior of $A$ in $X$ and by bd $(A)$ the boundary of $A$ in $X$. 
1. If $\hat{T}^{n} \hat{x}=\hat{x}$, then $T^{n} \pi \hat{x}=\pi \hat{x}$.

2. If $T^{n} x=x$ and if $x \in \operatorname{int}(\pi \hat{Z})$ for all $\hat{Z} \in \hat{\mathscr{Z}}$ such that $x \in \pi(\hat{Z})$, then there is a unique $\hat{x} \in \pi^{-1} x$ such that $\hat{T}^{n} \hat{x}=\hat{x}$.

3. If $T^{n} x=x$ and if $x \in \bigcup_{\hat{Z} \in \hat{\mathscr{Z}}}$ bd $(\pi(\hat{Z}))$, then there are at most four $\hat{x} \in \pi^{-1} x$ which are $\hat{T}$-periodic. Their periods are $n$ or $2 n$.

4. In particular, for a fixed $n$, the set $\left\{\hat{x}=\hat{T}^{n} \hat{x}\right\}$ is finite.

From Lemma 4.i of Hofbauer/Keller (1984), we obtain

Lemma 3.2. If $\hat{\eta} \in \hat{\mathscr{Z}}_{m}$ and $\hat{\eta} \subseteq \hat{T}^{m} \hat{\eta}$, then $\hat{\eta} \in \hat{\mathscr{Z}}_{m}^{2 m}$ and, furthermore, $\bigcup_{i=0}^{m} \hat{T}^{i} \hat{\eta} \subseteq \hat{\mathscr{D}}_{2 m}$.

The following lemma is crucial for the estimate in Sect. 5:

Lemma 3.3. Let $0<k \leqq m$. Given $\eta^{\prime} \in \mathscr{Z}_{k}$ and $C \in \mathscr{D}$, there are at most two $\hat{\eta} \in \hat{\mathscr{Z}}_{m}$ such that $\hat{\eta} \subseteq \hat{C}, \hat{T}^{m-k} \hat{\eta} \in \hat{\mathscr{Z}}_{k} \backslash \hat{\mathscr{Z}}_{m}^{2 m}$ and $\pi \hat{T}^{m-k} \hat{\eta} \subseteq \eta^{\prime}$.

Proof. Let $\hat{\eta} \in \hat{\mathscr{Z}}_{m}, \hat{\eta}=\hat{C} \cap \pi^{-1} \eta$, where $\hat{C} \in \hat{\mathscr{D}}, \eta \in \mathscr{Z}_{m}$. Write $\eta=\eta^{(1)} \cap T^{-(m-k)} \eta^{(2)}$, $\eta^{(1)} \in \mathscr{Z}_{m-k}, \eta^{(2)} \in \mathscr{Z}_{k}$, and define $\hat{\eta}^{(1)}:=\hat{C} \cap \pi^{-1} \eta^{(1)}$. Then

$$
\hat{\eta}=\hat{\eta}^{(1)} \cap \hat{T}^{-(m-k)} \pi^{-1} \eta^{(2)},
$$

whence

$$
\hat{T}^{m-k} \hat{\eta}=\hat{T}^{m-k} \hat{\eta}^{(1)} \cap \pi^{-1} \eta^{(2)}
$$

and

$$
\pi \hat{T}^{m-k} \hat{\eta}=T^{m-k}\left(C \cap \eta^{(1)}\right) \cap \eta^{(2)} .
$$

Now the assumption $\pi \hat{T}^{m-k} \hat{\eta} \subseteq \eta^{\prime} \in \mathscr{Z}_{k}$ implies $\eta^{(2)}=\eta^{\prime}$, and because of the hypothesis $\hat{T}^{m-k} \hat{\eta} \in \hat{\mathscr{Z}}_{k} \backslash \hat{\mathscr{Z}}_{k}^{2 m}$, we have

$$
\widehat{T}^{m-k} \hat{\eta}^{(1)} \in \hat{\mathscr{D}} \backslash \hat{\mathscr{D}}_{2 m},
$$

i.e.,

$$
T^{m-k}\left(C \cap \eta^{(1)}\right)=T^{m-k} \pi \hat{\eta}^{(1)}=\pi \hat{T}^{m-k} \hat{\eta}^{(1)} \in \mathscr{D} \backslash \mathscr{D}_{2 m} .
$$

As $T^{m-k} \eta^{(1)} \in \mathscr{D}_{m-k} \subseteq \mathscr{D}_{2 m}$, this implies $\eta^{(1)} \nsubseteq C$, and for a fixed interval $C$ there are at most two $\eta^{(1)} \in \mathscr{Z}_{m-k}$ with this property. Hence, given $C$ and $\eta^{\prime}$, there are at most two $\hat{\eta}=\hat{C} \cap \pi^{-1} \eta^{(1)} \cap \hat{T}^{-(m-k)} \pi^{-1} \eta^{\prime}$ satisfying the hypotheses of the lemma.

We need some more notations:

$\widehat{B V}$ denotes the space of function of bounded variation from $X^{\prime}$ to $\mathbb{C}$. We define $\pi_{*}: \widehat{B V} \rightarrow B V$ by

$$
\pi_{*} \hat{f}(x)=\sum_{\hat{x} \in \pi^{-1} x} \hat{f}(\hat{x}) .
$$

Observe that $\left\|\pi_{*} \hat{f}\right\|_{B V}=\operatorname{var}\left(\pi_{*} \hat{f}\right)+\sup \left|\pi_{*} \hat{f}\right| \leqq \sum_{C \in \mathscr{D}}\left(\operatorname{var}_{\hat{C}}(\hat{f})+\sup _{\hat{C}}|\hat{f}|\right)=\|\hat{f}\|_{\boldsymbol{B} V}$.

We associate to $g: X \rightarrow \mathbb{C}$ a weight function in the Markov extension, $\hat{g}: X^{\prime} \rightarrow \mathbb{C}$, $\hat{g}=g \circ \pi$. As $\pi X^{\prime}=X, \hat{\theta}=\lim _{n \rightarrow \infty}\left(\sup \left|\hat{g}_{n}\right|\right)^{1 / n}=\theta$. We denote by $\hat{\mathscr{L}}$ the transfer operator 
corresponding to $\hat{T}$ and $\hat{g}$. Since $(2.3)$ is satisfied for $\hat{g}, \hat{\mathscr{L}}$ maps $\widehat{B V}$ to $\widehat{B V}$ and

$$
\pi_{*} \circ \hat{\mathscr{L}}=\mathscr{L} \circ \pi_{*} .
$$

We end this section with the proof of Theorem 3 from the introduction: The claim is that $P(T, \log |g|) \geqq \log \rho(\mathscr{L})$ and that, if $g \geqq 0$, then equality holds. First we prove $\log \rho(\mathscr{L}) \leqq P(T, \log |g|)$ :

If $\theta<\rho(\mathscr{L})$, then let $\lambda$ be an eigenvalue of $\mathscr{L}$ with $|\lambda|=\rho(\mathscr{L})$. Suppose $\mathscr{L} f=\lambda f$, $0 \neq f \in B V$. Fix $x$ such that $f(x) \neq 0$. Then

$$
\left|\lambda^{m} f(x)\right|=\left|\mathscr{L}^{m} f(x)\right| \leqq \sum_{y \in T^{-m_{x}}}\left|g_{m}(y)\right||f(y)| \leqq \sup |f| \cdot \sum_{\eta \in \mathscr{Z}_{m}} \sup _{\eta}\left|g_{m}\right|
$$

whence $\log \rho(\mathscr{L})=\log |\lambda|=\limsup (1 / m) \log \left|\lambda^{m} f(x)\right| \leqq P(T, \log |g|)$. If $\theta=\rho(\mathscr{L})$, then $\log \rho(\mathscr{L})=\limsup _{m \rightarrow \infty}(1 / m) \log \sup \left|g_{m}\right| \leqq P(T, \log |g|)$.

For the reverse inequality, we assume first that $\inf g>0$. Then $\log g$ is continuous, and it follows from Theorem 1.9 of Walters (1976) that

$$
P(T, \log (g))=\limsup _{m \rightarrow \infty} \frac{1}{m} \log \sum_{\eta \in \mathscr{Z}_{m}} \inf \left(g_{m}\right) .
$$

Now fix some $z_{D} \in D$ for each $D \in \mathscr{D}$. As $T^{m} \eta \in \mathscr{D}_{m}$ for each $\eta \in \mathscr{Z}_{m}$, we have

$$
\sum_{\eta \in \mathscr{Z}_{m}} \inf \left(g_{m}\right) \leqq \sum_{D \in \mathscr{D}_{m}} \sum_{y \in T^{-m_{z_{D}}}} g_{m}(y)=\sum_{D \in \mathscr{D}_{m}}\left(\mathscr{L}^{m} 1\right)\left(z_{D}\right) \leqq \text { const } \cdot m \cdot\left\|\mathscr{L}^{m} 1\right\|_{B V},
$$

whence $P(T, \log (g)) \leqq \log \rho(\mathscr{L})$.

If only $g \geqq 0$ is assumed, we approximate $g$ from above by $g \vee \varepsilon=\sup (g, \varepsilon)$ $(\varepsilon>0)$. As $g \vee \varepsilon \in B V$ and $g \vee \varepsilon \geqq \varepsilon>0, P(T, \log (g \vee \varepsilon))=\log \rho\left(\mathscr{L}_{\varepsilon}\right)$, where $\mathscr{L}_{\varepsilon}$ is the transfer operator obtained from $\mathscr{L}$ by replacing $g$ by $g \vee \varepsilon$. As $g \leqq g \vee \varepsilon$, $P(T, \log (g)) \leqq P(T, \log (g \vee \varepsilon))=\log \rho\left(\mathscr{L}_{\varepsilon}\right)$, and we have to show that $\liminf _{\varepsilon \rightarrow 0} \rho\left(\mathscr{L}_{\varepsilon}\right) \leqq$ $\rho(\mathscr{L})$. By the upper semicontinuity of the spectrum of bounded operators on Banach spaces (see Kato (1976), Chapter IV, Remark 3.3), it suffices to prove that $\lim _{\varepsilon \rightarrow 0}\left\|\mathscr{L}_{\varepsilon}-\mathscr{L}\right\|_{B V}=0$. But $\mathscr{L}_{\varepsilon}-\mathscr{L}$ is the transfer operator for the weight function $g \vee \varepsilon-g=(\varepsilon-g) \cdot \chi_{\{g \leqq \varepsilon\}}$, whence, by Lemma 2.2 (remember that $\left.\mathscr{I}=\{X\}\right)$ :

$$
\left\|\mathscr{L}_{\varepsilon}-\mathscr{L}\right\|_{B V} \leqq 3 \cdot(\operatorname{var}(g \vee \varepsilon-g)+\varepsilon \cdot \#(\mathscr{Z})),
$$

and we can finish the proof of the theorem by showing $\lim _{\varepsilon \rightarrow 0} \operatorname{var}(g \vee \varepsilon-g)=0$ :

As $\lim _{\varepsilon \rightarrow 0} g(x) \vee \varepsilon=g(x)$ for all $x \in X, \lim _{\varepsilon \rightarrow 0} \operatorname{var}(g \vee \varepsilon) \geqq \operatorname{var}(g)$. As

$$
|g(x)-g(y)|=|g(x) \vee \varepsilon-g(y) \vee \varepsilon|+|g(x) \vee \varepsilon-g(x)-g(y) \vee \varepsilon+g(y)|
$$

for all $x, y \in X, \operatorname{var}(g)=\operatorname{var}(g \vee \varepsilon)+\operatorname{var}(g \vee \varepsilon-g)$. Hence, $\lim _{\varepsilon \rightarrow 0} \operatorname{var}(g \vee \varepsilon-g)=$ $\operatorname{var}(g)-\lim _{\varepsilon \rightarrow 0} \operatorname{var}(g \vee \varepsilon) \leqq 0$.

\section{Proof of Theorem 2}

Throughout this section we assume (1.4), i.e. $\mathscr{Z}$ is a generating partition for $T$. For $m>0$, let $\zeta_{m}=\sum_{x=T^{m} x} g_{m}(x)$ and $\hat{\zeta}_{m}=\sum_{\hat{x}=\hat{T}^{m_{\hat{x}}}} \hat{g}_{m}(\hat{x})$. The zeta functions for $(T, g)$ and 
$(\hat{T}, \hat{g})$ are defined by:

$$
\zeta(z)=\exp \sum_{m=1}^{\infty} \frac{z^{m}}{m} \zeta_{m}, \quad \hat{\zeta}(z)=\exp \sum_{m=1}^{\infty} \frac{z^{m}}{m} \hat{\zeta}_{m} .
$$

Lemma 4.1. $\zeta(z) / \hat{\zeta}(z)$ is analytic and nonzero in $\left\{|z|<\theta^{-1}\right\}$.

This follows from Lemma 3.1 as in Hofbauer (1985).

For each $m>0$ and each $\hat{\eta} \in \hat{\mathscr{Z}}_{m}$, fix $\hat{x}_{\hat{\eta}} \in \hat{\eta}$ in such a way that $\hat{T}^{x} \hat{x}_{\hat{\eta}}=\hat{x}_{\hat{\eta}}$ if $\hat{\eta} \subseteq \hat{T}^{m} \hat{\eta}$ and arbitrary otherwise. (Note that the Markov property implies that $\hat{\eta}$ and $\hat{T}^{m} \hat{\eta}$ are disjoint if $\hat{\eta} \nsubseteq \hat{T}^{m} \hat{\eta}$.) Let $\hat{\mathscr{A}}_{m}=\left\{\hat{\eta} \in \hat{\mathscr{Z}}_{m}: \pi \hat{\eta} \in \mathscr{Z}_{m}\right\}$ and define for $\hat{f}$ in $\hat{B V}$

$$
\hat{\alpha}_{m} \hat{f}=\sum_{\hat{\eta} \in \hat{A}_{m}} \hat{f}\left(\hat{x}_{\hat{\eta}}\right) \chi_{\hat{\eta}} \text {. }
$$

As each $\hat{D}$ in $\hat{\mathscr{D}}$ is an interval, only those two $\hat{\eta} \subseteq \hat{D}$ which contain the endpoints of $\hat{D}$ may fail to satisfy $\pi \hat{\eta} \in \mathscr{Z}_{m}$, hence, $L$ as in (2.4) is at most 2 .

Lemma 4.2. For each bounded linear $\hat{\phi}: \widehat{B V} \rightarrow \widehat{B V}$ and each $m>0, \hat{\phi} \hat{\mathscr{L}}^{m} \hat{\alpha}_{m}$ is a finite rank operator and

$$
\operatorname{tr}\left(\hat{\phi} \hat{\mathscr{L}}^{m} \hat{\alpha}_{m}\right)=\sum_{\hat{\eta} \in \hat{\mathscr{A}}_{m}}\left(\hat{\phi} \hat{\mathscr{L}}^{m} \chi_{\dot{\eta}}\right)\left(\hat{x}_{\hat{\eta}}\right)
$$

where the right hand-side converges absolutely.

Proof. For $\hat{f}$ in $\widehat{B V}$, we have

where $F_{\eta}(\hat{f})=\sum_{\substack{\hat{\eta} \in \hat{\mathbb{P}}_{m} \\ \pi \hat{\eta}=\eta}} \hat{f}\left(\hat{x}_{\hat{\eta}}\right)$ and

$$
\hat{\phi} \hat{\mathscr{L}}^{m} \hat{\alpha}_{m} \hat{f}=\sum_{\hat{\eta} \in \hat{\mathscr{A}}_{m}} \hat{f}\left(\hat{x}_{\hat{\eta}}\right) \hat{\phi} \hat{\mathscr{L}}^{m} \chi_{\hat{\eta}}=\sum_{\eta \in \mathscr{Z}_{m}} F_{\eta}(\hat{f}) \cdot \hat{\phi} \hat{\mathscr{L}}^{m} \chi_{e(\eta)},
$$

$$
\left|F_{\eta}(\hat{f})\right| \leqq \sum_{\substack{\hat{\eta} \in \hat{\mathscr{Y}}_{m} \\ \pi \hat{\eta}=\eta}}\left|\hat{f}\left(\hat{x}_{\hat{\eta}}\right)\right| \leqq\|\hat{f}\|_{\infty} \leqq\|\hat{f}\|_{\hat{B V}^{-}} .
$$

As $\mathscr{Z}_{m}$ is finite, $\hat{\phi} \hat{\mathscr{L}}^{m} \hat{\alpha}_{m}$ has finite rank. Now

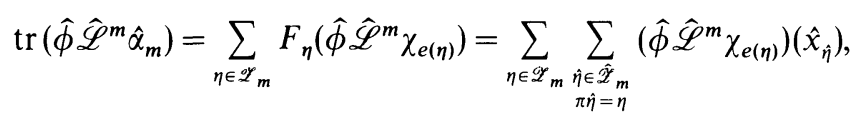

and the sum converges absolutely, because $\mathscr{Z}_{m}$ is finite and

$$
\sum_{\substack{\hat{\eta} \in \hat{\mathscr{Y}}_{m} \\ \pi \hat{\eta}=\eta}}\left|\hat{\phi} \hat{\mathscr{L}}^{m} \chi_{e(\eta)}\right|\left(\hat{x}_{\hat{\eta}}\right) \leqq\left\|\hat{\phi} \hat{\mathscr{L}}^{m} \chi_{e(\eta)}\right\|_{\infty}<\infty
$$

Hence $\operatorname{tr}\left(\hat{\phi} \hat{\mathscr{L}}^{m} \hat{\alpha}_{m}\right)=\sum_{\hat{\eta} \in \hat{\mathscr{A}}_{m}}\left(\hat{\phi} \hat{\mathscr{L}}^{m} \chi_{\hat{\eta}}\right)\left(\hat{x}_{\hat{\eta}}\right)$ converges absolutely.

In particular, $\hat{\mathscr{L}}$ has the spectral decomposition

$$
\hat{\mathscr{L}}^{m}=\sum_{i=1}^{\hat{N}(\Theta)} \hat{\lambda}_{i}^{m} \hat{\mathscr{P}}_{i} \hat{\Lambda}_{i}^{m}+\hat{\mathscr{P}} \hat{\mathscr{L}}^{m},
$$

analogous to (2.6) and (1.1).

Lemma 4.3. Let $|\lambda|>\theta$. $\lambda$ is an eigenvalue of $\mathscr{L}$ of multiplicity $d$ if and only if it is an eigenvalue of $\hat{\mathscr{L}}$ of multiplicity $d$. 
Proof. Suppose $\lambda$ is an eigenvalue of $\mathscr{L}$ with multiplicity $d$. In order to prove that it is an eigenvalue of $\hat{\mathscr{L}}$ with multiplicity at least $d$, it suffices to show that if $(\mathscr{L}-\lambda \text { Id })^{k} f=0$ for some $f \in B V$ and $k>0$ then there is $\hat{f} \in \hat{B V}$ with $\pi_{*} \hat{f}=f$ and $(\hat{\mathscr{L}}-\lambda \text { Id })^{\hat{k}} \hat{f}=0$ for some $\hat{k}>0$. But this is Proposition 7.1.bis together with Remark 7.2 of Keller (1986).

Suppose conversely that $\lambda$ is an eigenvalue of $\hat{\mathscr{L}}$ with multiplicity $d$. In order to show that it is an eigenvalue of $\mathscr{L}$ with multiplicity at least $d$, it suffices to show that if $(\hat{\mathscr{L}}-\lambda \mathrm{Id})^{k} \hat{f}=0$ for some $0 \neq \hat{f} \in \widehat{B V}$ and $k>0$, then $\pi_{*} \hat{f} \neq 0$ and $(\mathscr{L}-\lambda \text { Id })^{k} \pi_{*} \hat{f}=0$. The latter follows from (3.1) and we must only prove $\pi_{*} \hat{f} \neq 0$.

So suppose $\pi_{*} \hat{f}=0$. We consider the case $k=1$ first, i.e., we assume $\hat{f}=\lambda^{-1} \hat{\mathscr{L}} \hat{f}$. Fix $\hat{x}=(x, D) \in X^{\prime}$. Then, for all $m>0$,

$$
\hat{f}(\hat{x})=\sum_{\hat{y} \in \hat{T}^{-m_{\hat{x}}}} \lambda^{-m} \hat{g}_{m}(\hat{y}) \hat{f}(\hat{y})=\sum_{y \in T^{-m_{x}}} \lambda^{-m} g_{m}(y) \cdot S_{m, D}(y),
$$

where

$$
S_{m, D}(y)=\sum_{\substack{(y, C) \text { s.th. } \\ T^{m}\left(C \cap \eta_{m}[y]\right)=D}} \hat{f}(y, C) .
$$

If $T^{m} \eta_{m}[y]=D$, then $T^{m}\left(C \cap \eta_{m}[y]\right)=D$ if and only if $\eta_{m}[y] \subseteq C$. Hence, for such $y$,

$$
S_{m, D}(y)=\sum_{\hat{y} \in \pi^{-1} y} \hat{f}(\hat{y})-\sum_{\substack{(y, C) s \text { th. } \\ \eta_{m}[y] \notin C}} \hat{f}(y, C) .
$$

If $T^{m} \eta_{m}[y] \neq D$, i.e., if $D$ is a proper subset of $T^{m} \eta_{m}[y]$, then $T^{m}\left(C \cap \eta_{m}[y]\right)=D$ implies $\eta_{m}[y] \nsubseteq C$.

In any case

$$
\left|S_{m, D}(y)\right| \leqq \sum_{\substack{(y, C) \text { s.th. } \\ \eta_{m}[y] \nsubseteq C}}|\widehat{f}(y, C)|,
$$

because $\sum_{\hat{y} \in \pi^{-1} y} \hat{f}(\hat{y})=\pi_{*} \hat{f}(y)=0$. Therefore, for large $m$,

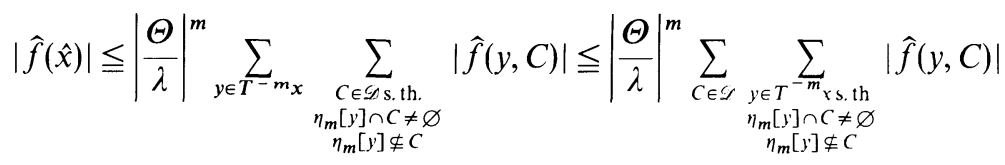

$$
\begin{aligned}
& \leqq\left|\frac{\Theta}{\lambda}\right|^{m} \sum_{C \in \mathscr{L}} 2 \cdot \sup _{\hat{C}}|\hat{f}| \leqq\left|\frac{\Theta}{\lambda}\right|^{m} \cdot 2\|\hat{f}\|_{\infty} \text {. }
\end{aligned}
$$

As $|\lambda|>\theta$, we may assume $\theta<\Theta<|\lambda|$, whence $|\hat{f}(\hat{x})|=0$ as $m \rightarrow \infty$.

The case $k>1$ is easily reduced to the case $k=1$ : Suppose $(\hat{\mathscr{L}}-\lambda \text { Id })^{k} \hat{f}=0$ and $\pi_{*} \hat{f}=0$. Let $\hat{f}_{1}=(\hat{\mathscr{L}}-\lambda \text { Id })^{k-1} \hat{f}$. Then $(\hat{\mathscr{L}}-\lambda$ Id $) \hat{f}_{1}=0$ and $\pi_{*} \hat{f}_{1}=0$ in view of (3.1). Hence $\hat{f}_{1}=0$ by the case $k=1$ and $\hat{f}=0$ follows by induction.

Remark. We would like to mention that this proof can be adapated to the systems investigated in Sect. 7 of Keller (1986).

Because of Lemmas 4.1 and 4.3, and Proposition 1.1, it suffices to prove Theorem 2 for $\hat{\mathscr{L}}$ and $\hat{\zeta}$. As a matter of fact, we shall prove the more detailed 
Theorem 4.4. Assume (2.1)-(2.3) and (1.4), then for each $\Theta>\theta, \hat{\zeta}(z) \cdot \prod_{i=1}^{\hat{N}(\Theta)}\left(1-\hat{\lambda}_{i} z\right)^{\mathrm{rank}\left(\hat{\mathscr{P}}_{i}\right)}$ is analytic and nonzero in $\left\{|z|<\Theta^{-1}\right\}$.

Observe that $\operatorname{rank}\left(\widehat{\mathscr{P}}_{i}\right)=\operatorname{tr}\left(\widehat{\mathscr{P}}_{i}\right)$. As

$$
\begin{aligned}
\prod_{i=1}^{\hat{N}(\theta)}\left(1-\hat{\lambda}_{i} z\right)^{\operatorname{tr}\left(\hat{\mathscr{P}}_{i}\right)} & =\exp \sum_{i=1}^{\hat{N}(\Theta)} \sum_{m=1}^{\infty}-\frac{z^{m}}{m} \hat{\lambda}_{i}^{m} \operatorname{tr}\left(\hat{\mathscr{P}}_{i}\right) \\
& =\exp \sum_{m=1}^{\infty}-\frac{z^{m}}{m} \operatorname{tr}\left(\sum_{i=1}^{\hat{N}(\Theta)} \hat{\lambda}_{i}^{m} \hat{\mathscr{P}}_{i} \hat{\Lambda}_{i}^{m}\right) \quad \text { by }(2.7) \\
& =\exp \sum_{m=1}^{\infty}-\frac{z^{m}}{m} \operatorname{tr}\left(\sum_{i=1}^{\hat{N}(\Theta)} \hat{\mathscr{P}}_{i} \hat{\mathscr{L}}^{m}\right)
\end{aligned}
$$

Theorem 4.4 will be proved if we can show that

$$
\left|\hat{\zeta}_{m}-\operatorname{tr}\left(\sum_{i=1}^{\hat{N}(\Theta)} \hat{\mathscr{P}}_{i} \hat{\mathscr{L}}^{m}\right)\right| \leqq \text { const } \cdot \Theta^{m} \text {. }
$$

In the course of the proof, estimates by terms of the form $m \cdot \Theta^{m}$ or $(1+\varepsilon)^{m} \cdot \Theta^{m}$ will occur. In order to simplify our notation, we shall replace them tacitly by const $\cdot \Theta^{m}$. This is possible because $\Theta>\theta$ is arbitrary.

Let $\hat{\zeta}_{m}^{(0)}=\sum_{\substack{\hat{\eta} \in \hat{A}_{m} \\ \hat{\eta} \subseteq \hat{T}^{m} \hat{\eta}}} \hat{g}_{m}\left(\hat{x}_{\hat{\eta}}\right)$ and $\hat{\zeta}_{m}^{(1)}=\hat{\zeta}_{m}-\hat{\zeta}_{m}^{(0)}$. As $\hat{\zeta}_{m}^{(0)}$ is a finite sum (see Lemma 3.1), and as $\hat{\mathscr{L}}^{m} \chi_{\hat{\eta}}(\hat{y})=0$ for $\hat{y} \notin \hat{T}^{m} \hat{\eta}$, we have in view of Lemma 4.2

$$
\begin{aligned}
& \hat{\zeta}_{m}^{(0)}=\sum_{\substack{\hat{\eta} \in \hat{A}_{m} \\
\hat{\eta} \subseteq \hat{T}^{m}}}\left(\hat{g}_{m} \cdot \chi_{\hat{\eta}}\right) \circ \hat{T}_{\hat{\eta}}^{-m}\left(\hat{x}_{\hat{\eta}}\right)=\sum_{\substack{\hat{\eta} \in \hat{A}_{m}^{m} \\
\hat{\eta} \subseteq \hat{T}^{m} \hat{\eta}}}\left(\hat{\mathscr{L}}^{m} \chi_{\hat{\eta}}\right)\left(\hat{x}_{\hat{\eta}}\right) \\
& =\sum_{\hat{\eta} \in \hat{\mathbb{A}}_{m}}\left(\hat{\mathscr{L}}^{m} \chi_{\hat{\eta}}\right)\left(\hat{x}_{\hat{\eta}}\right)=\operatorname{tr}\left(\hat{\mathscr{L}}^{m} \hat{\alpha}_{m}\right) .
\end{aligned}
$$

For $\widehat{\zeta}_{m}^{(1)}$ we have the estimate (observe Lemma 3.2)

$$
\begin{aligned}
\left|\hat{\zeta}_{m}^{(1)}\right| & \leqq \sum_{\substack{\hat{\eta} \in \hat{\mathscr{P}}_{m} \backslash \hat{\mathbb{P}}_{m} \\
\hat{\eta} \subseteq \hat{T}^{m}}}\left|\hat{g}_{m}\left(\hat{x}_{\hat{\eta}}\right)\right| \leqq \sum_{\hat{\eta}_{\hat{\eta}} \in \hat{\mathscr{P}}_{m}^{2 m} \backslash \hat{\mathscr{A}}_{m}}\left|\hat{g}_{m}\left(\hat{x}_{\hat{\eta}}\right)\right| \\
& \leqq 2 \cdot \#\left(\mathscr{D}_{2 m}\right) \cdot \sup \left|\hat{g}_{m}\right| \leqq \mathrm{const} \cdot \Theta^{m} .
\end{aligned}
$$

So (4.2) will follow if we show that

$$
\sum_{i=1}^{\hat{N}(\Theta)}\left|\operatorname{tr}\left(\hat{\mathscr{P}}_{i}\left(\hat{\mathscr{L}}^{m} \hat{\alpha}_{m}-\hat{\mathscr{L}}^{m}\right)\right)\right|+\left|\operatorname{tr}\left(\hat{\mathscr{P}} \hat{\mathscr{L}}^{m} \hat{\alpha}_{m}\right)\right| \leqq \text { const } \cdot \Theta^{m}
$$

By Proposition 2.7,

$$
\begin{aligned}
\sum_{i=1}^{\hat{N}(\Theta)}\left|\operatorname{tr}\left(\hat{\mathscr{P}}_{i}\left(\hat{\mathscr{L}}^{m} \hat{\alpha}_{m}-\hat{\mathscr{L}}^{m}\right)\right)\right| & \leqq \sum_{i=1}^{\hat{N}(\Theta)} \operatorname{rank}\left(\widehat{\mathscr{P}}_{i}\right) \cdot\left\|\hat{\mathscr{P}}_{i}\right\|_{\widehat{B V}}\left\|\hat{\mathscr{L}}^{m} \hat{\alpha}_{m}-\hat{\mathscr{L}}^{m}\right\|_{\widehat{B V}} \\
& \leqq \text { const } \cdot \Theta^{m}
\end{aligned}
$$

and the proof of (4.2) will be finished in the next section by showing that

$$
\left|\operatorname{tr}\left(\hat{\mathscr{P}} \hat{\mathscr{L}}^{m} \hat{\alpha}_{m}\right)\right| \leqq \text { const } \cdot \Theta^{m} \text {. }
$$


In order to do this, we will need more sophisticated estimates and make use of a telescoping trick of Haydn (1987).

\section{The Bound for $\left|\operatorname{tr}\left(\hat{\mathscr{P}} \hat{\mathscr{L}}^{m} \hat{\alpha}_{m}\right)\right|$}

In this section, we prove estimate (4.6) for $\hat{\zeta}_{m}^{(2)}=\operatorname{tr}\left(\hat{\mathscr{P}} \hat{\mathscr{L}}^{m} \hat{\alpha}_{m}\right)$ under the additional assumption (1.4).

For each $\hat{C} \in \hat{\mathscr{D}}$, we fix some $\hat{y}_{\hat{C}} \in \hat{C}$. If $\hat{\eta} \in \hat{\mathscr{Z}}_{k}$ and $\hat{T}^{k} \hat{\eta}=\hat{C}$, we denote by $\hat{y}_{\hat{\eta}}$ the unique $\hat{T}^{k}$ preimage of $\hat{y}_{\hat{C}}$ in $\hat{\eta}$.

For $\hat{\eta} \in \hat{\mathscr{Z}}_{k}$, define

$$
\hat{Y}_{\hat{\eta}}= \begin{cases}\hat{\mathscr{L}}^{k} \chi_{\hat{\eta}}-\hat{g}\left(\hat{y}_{\hat{\eta}}\right) \cdot \hat{\mathscr{L}}^{k-1} \chi_{\hat{T} \hat{\eta}} & \text { if } \quad k \geqq 2, \\ \hat{\mathscr{L}} \chi_{\hat{\eta}} & \text { if } \quad k=1 .\end{cases}
$$

Observe that $\hat{T} \hat{y}_{\hat{\eta}}=\hat{y}_{\hat{T} \hat{\eta}}$. Hence, for $\hat{\eta} \in \hat{\mathscr{Z}}_{k}$ and $k \geqq 2$,

$$
\hat{Y}_{\hat{\eta}}=\hat{\mathscr{L}}^{k-1} \chi_{\hat{T} \hat{\eta}} \cdot\left(\hat{g}^{\circ} \hat{T}_{\hat{\eta}}^{-k}-\hat{g}\left(\hat{y}_{\hat{\eta}}\right)\right)=\chi_{\hat{T}^{k} \hat{\eta}} \cdot \hat{g}_{k-1} \circ \hat{T}_{\hat{T} \hat{\eta}}^{-(k-1)} \cdot\left(\hat{g}^{\circ} \hat{T}_{\hat{\eta}}^{-k}-\hat{g}\left(\hat{y}_{\hat{\eta}}\right)\right),
$$

so that

$$
\operatorname{var}_{\hat{T}^{k} \hat{\eta}}\left(\hat{Y}_{\hat{\eta}}\right) \leqq \operatorname{var}_{\hat{T}_{\hat{\eta}}}\left(\hat{g}_{k-1}\right) \cdot \operatorname{var}_{\hat{\eta}}(\hat{g})+\sup \left|\hat{g}_{k-1}\right| \cdot \operatorname{var}_{\hat{\eta}}(\hat{g})
$$

and

$$
\sup \left|\hat{Y}_{\hat{\eta}}\right| \leqq \sup \left|\hat{g}_{k-1}\right| \cdot \operatorname{var}_{\hat{\eta}}(\hat{g})
$$

Therefore

$$
\begin{aligned}
\left\|\hat{Y}_{\hat{\eta}}\right\|_{\widehat{B V}} & =\operatorname{var}\left(\hat{Y}_{\hat{\eta}}\right)+\left\|\hat{Y}_{\hat{\eta}}\right\|_{\infty} \leqq \operatorname{var}_{\hat{T}^{k} \hat{\eta}}\left(\hat{Y}_{\hat{\eta}}\right)+3 \cdot \sup \left|\hat{Y}_{\hat{\eta}}\right| \\
& \leqq \operatorname{var}_{\hat{\eta}}(\hat{g}) \cdot\left(\operatorname{var}_{\hat{T}_{\hat{\eta}}}\left(\hat{g}_{k-1}\right)+4 \cdot \sup \left|\hat{g}_{k-1}\right|\right) .
\end{aligned}
$$

If $\pi \hat{\eta} \subseteq \eta \in \mathscr{Z}_{k}$, this yields

$$
\left\|\hat{Y}_{\hat{\eta}}\right\|_{\widehat{B V}} \leqq \operatorname{var}_{\eta}(g) \cdot\left(\operatorname{var}_{T \eta}\left(g_{k-1}\right)+4 \sup \left|g_{k-1}\right|\right)=: v(\eta, k) .
$$

Note also that in view of Corollary 2.4,

$$
\sum_{\eta \in \mathscr{Z}_{k}} v(\eta, k) \leqq C \cdot \Theta^{k-1} \cdot \operatorname{var}(g)
$$

We now give the formal computation used to bound $\left|\widehat{\zeta}_{m}^{(2)}\right|$. By Lemma 4.2,

$$
\begin{aligned}
\hat{\zeta}_{m}^{(2)}= & \operatorname{tr}\left(\hat{\mathscr{P}} \hat{\mathscr{L}}^{m} \hat{\alpha}_{m}\right)=\sum_{\hat{\eta} \in \hat{\mathscr{A}}_{m}}\left(\hat{\mathscr{P}} \hat{\mathscr{L}}^{m} \chi_{\hat{\eta}}\right)\left(\hat{x}_{\hat{\eta}}\right) & \left(=: \gamma_{m}^{(0)}\right) \\
= & \sum_{\hat{\eta} \in \hat{\mathscr{X}}_{m}}\left(\hat{\mathscr{P}} \hat{\mathscr{L}}^{m} \chi_{\hat{\eta}}\right)\left(\hat{x}_{\hat{\eta}}\right) & \left(=: \gamma_{m}^{(1)}\right) \\
& -\sum_{\hat{\eta} \in \hat{\mathscr{X}}_{m} \backslash \hat{\mathscr{A}}_{m}}\left(\hat{\mathscr{P}} \hat{\mathscr{L}}^{m} \chi_{\hat{\eta}}\right)\left(\hat{x}_{\hat{\eta}}\right) & \left(=: \gamma_{m}^{(2)}\right) .
\end{aligned}
$$

Now

$$
\gamma_{m}^{(1)}=\sum_{\hat{\eta} \in \hat{\mathscr{X}}_{m}}\left(\hat{\mathscr{P}} \hat{\mathscr{L}}^{m} \chi_{\hat{\eta}}\right)\left(\hat{x}_{\hat{\eta}}\right)=\sum_{\hat{\eta} \in \hat{\mathscr{X}}_{m}} \sum_{k=0}^{m-1} \hat{g}_{k}\left(\hat{y}_{\hat{\eta}}\right) \hat{\mathscr{P}} \hat{Y}_{\hat{T}^{k}}\left(\hat{x}_{\hat{\eta}}\right)
$$




$$
\begin{array}{rlr}
= & \sum_{\hat{\eta} \in \hat{\mathscr{P}}_{m}} \sum_{k=0}^{m-1} \hat{g}_{k}\left(\hat{y}_{\hat{\eta}}\right)\left(\hat{\mathscr{P}} \hat{Y}_{\hat{T}^{k}}\left(\hat{x}_{\hat{\eta}}\right)-\hat{\mathscr{P}} \hat{Y}_{\hat{T}^{k} \hat{\eta}}\left(\hat{y}_{\hat{\eta}}\right)\right) & \left(=: \gamma_{m}^{(3)}\right) \\
& +\sum_{\hat{\eta} \in \hat{\mathscr{Y}}_{m}} \sum_{k=0}^{m-1} \hat{g}_{k}\left(\hat{y}_{\hat{\eta}}\right) \hat{\mathscr{P}} \hat{Y}_{\hat{T}^{k}}\left(\hat{y}_{\hat{\eta}}\right), & \left(=: \gamma_{m}^{(4)}\right),
\end{array}
$$

where we use the notational convention $\hat{g}_{0} \equiv 1$. Finally

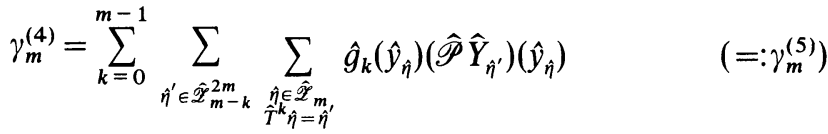



The result of this section follows from:

\section{Lemma 5.1.}

1. $\gamma_{m}^{(4)}$ converges absolutely,

2. $\gamma_{m}^{(2)}$ converges absolutely and $\left|\gamma_{m}^{(2)}\right| \leqq$ const $\cdot \Theta^{m}$,

3. $\gamma_{m}^{(3)}$ converges absolutely and $\left|\gamma_{m}^{(3)}\right| \leqq$ const $\cdot \Theta^{m}$,

4. $\left|\gamma_{m}^{(5)}\right| \leqq$ const $\cdot \Theta^{m}$

5. $\left|\gamma_{m}^{(6)}\right| \leqq$ const $\cdot \Theta^{m}$.

(All bounds are uniform in $m>0$ ).

Indeed, the absolute convergence of $\gamma_{m}^{(3)}$ and $\gamma_{m}^{(4)}$ implies that of $\gamma_{m}^{(1)}$ and shows that all equalities of the sequence (5.4)-(5.6) are correct. Thus, we only need to use

$$
\left|\widehat{\zeta}_{m}^{(2)}\right| \leqq\left|\gamma_{m}^{(2)}\right|+\left|\gamma_{m}^{(3)}\right|+\left|\gamma_{m}^{(5)}\right|+\left|\gamma_{m}^{(6)}\right|
$$

to obtain the desired inequality.

Proof of Lemma 5.1. In the proof, we will often use the decomposition $\widehat{\mathscr{P}}=\mathrm{Id}-\sum_{i=1}^{\hat{N}(\Theta)} \widehat{\mathscr{P}}_{i}$ and the fact that for each $\hat{\mathscr{P}}_{i}$ there are $\hat{\varphi}_{i j} \in \widehat{B V}$ and linear functionals $\hat{\psi}_{i j}\left(j=1, \ldots, \operatorname{rank}\left(\hat{\mathscr{P}}_{i}\right)=: d_{i}\right)$ such that $\hat{\mathscr{P}}_{i}(\hat{f})=\sum_{j=1}^{d_{i}} \hat{\psi}_{i j}(\hat{f}) \hat{\varphi}_{i j}$.

1. We have

$$
\begin{aligned}
& \sum_{\hat{\eta} \in \hat{\mathscr{Y}}_{m}} \sum_{k=0}^{m-1}\left|\hat{g}_{k}\left(\hat{y}_{\hat{\eta}}\right)\right|\left|\hat{\mathscr{P}} \hat{Y}_{\hat{T}^{k}}\left(\hat{y}_{\hat{\eta}}\right)\right| \\
& =\sum_{k=0}^{m-1} \sum_{\substack{\hat{\eta}^{\prime} \in \hat{\mathscr{Y}}_{m-k} \\
\hat{T}_{m} \in \hat{\mathscr{P}}_{m,}}}\left|\hat{g}_{k}\left(\hat{y}_{\hat{\eta}}\right)\right|\left|\hat{\mathscr{P}} \hat{Y}_{\hat{\eta}^{\prime}}\left(\hat{y}_{\hat{\eta}}\right)\right| \\
& =\sum_{k=0}^{m-1} \sum_{\eta^{\prime} \in \hat{\mathscr{Y}}_{m-k}}\left(|\hat{\mathscr{L}}|^{k}\left|\hat{\mathscr{P}} \hat{Y}_{\hat{\eta}^{\prime}}\right|\right)\left(\hat{y}_{\hat{\eta}^{\prime}}\right) \\
& \leqq \sum_{k=0}^{m-1} \sum_{\hat{\eta}^{\prime} \in \hat{\mathscr{Y}}_{m-k} \backslash \hat{\mathscr{X}}_{m-k}^{2 m}} \sum_{i=1}^{\hat{N}(\Theta)} \sum_{j=1}^{d_{i}}\left(|\hat{\mathscr{L}}|^{k}\left|\hat{\varphi}_{i j}\right|\right)\left(\hat{y}_{\hat{\eta}^{\prime}}\right)\left\|\hat{\psi}_{i j}\right\|\left\|\hat{Y}_{\hat{\eta}^{\prime}}\right\|_{B V} \quad\left(=: \beta_{m}^{(1)}\right) \\
& +\sum_{k=0}^{m-1} \sum_{\eta^{\prime} \in \hat{\mathscr{Y}}_{m-k}^{2 m}}\left(|\hat{\mathscr{L}}|^{k}\left|\hat{\mathscr{P}} \hat{Y}_{\hat{\eta}^{\prime}}\right|\right)\left(\hat{y}_{\hat{\eta}^{\prime}}\right) \\
& \left(=: \beta_{m}^{(2)}\right) \text {. }
\end{aligned}
$$


Remark. In the last inequality, we used the fact that $\left.\left(|\hat{\mathscr{L}}|^{k}\left|\hat{Y}_{\hat{\eta}^{\prime}}\right|\right)\right|_{\hat{\eta}^{\prime}} \not \equiv 0$ if and only if $\left|\left(Y_{\hat{\eta}^{\prime}}\right)\right|_{\hat{\eta}} \neq \equiv 0$ for some $\hat{\eta} \in \hat{\mathscr{Z}}_{m}$ with $\hat{T}^{k} \hat{\eta}=\hat{\eta}^{\prime}$. But this is possible only if $\hat{T}^{m-k} \hat{\eta}^{\prime} \supseteq \hat{\eta}$, i.e., if $\hat{T}^{m} \hat{\eta} \supseteq \hat{\eta}$, i.e., if $\hat{\eta} \in \hat{\mathscr{Z}}_{m}^{2 m}$ and also $\hat{\eta}^{\prime} \in \hat{\mathscr{Z}}_{m-k}^{2 m}$ by Lemma 3.2. Therefore the term corresponding to the Id part in the decomposition $\widehat{\mathscr{P}}=\mathrm{Id}-\sum_{i=1}^{\hat{N}(\Theta)} \hat{\mathscr{P}}_{i}$ is zero in the
first sum.

Now, by (5.2) and (5.3):

$$
\begin{aligned}
\beta_{m}^{(1)} & \leqq \sum_{k=0}^{m-1} \sum_{i=1}^{\hat{N}(\Theta)} \sum_{j=1}^{d_{i}}\left\|\hat{\psi}_{i j}\right\| \sum_{\eta^{\prime} \in \mathscr{X}_{m-k}} v\left(\eta^{\prime}, m-k\right) \sum_{\substack{\hat{\eta}^{\prime} \in \hat{\mathscr{X}}_{m-k} \\
\pi \hat{\eta}^{\prime} \subseteq \hat{\eta}^{k}}}\left(|\hat{\mathscr{L}}|^{k}\left|\hat{\varphi}_{i j}\right|\right)\left(\hat{y}_{\hat{\eta}^{\prime}}\right) \\
& \leqq \sum_{k=0}^{m-1} \sum_{i=1}^{\hat{N}(\Theta)} \sum_{j=1}^{d_{i}}\left\|\hat{\psi}_{i j}\right\| \cdot \operatorname{const} \cdot \Theta^{m-k-1} \cdot \operatorname{var}(g) \cdot\left\||\hat{\mathscr{L}}|^{k}\left|\hat{\varphi}_{i j}\right|\right\|_{\boldsymbol{B V}}<\infty,
\end{aligned}
$$

and $\beta_{m}^{(2)}<\infty$ as \# $\hat{\mathscr{Z}}_{m-k}^{2 m} \leqq 2 m \cdot \# \mathscr{Z}_{m-k}<\infty$.

2. We again use the decomposition of $\hat{\mathscr{P}}$ :

$$
\begin{aligned}
& \left|\gamma_{m}^{(2)}\right| \leqq \sum_{\hat{\eta} \in \hat{\mathscr{X}}_{m} \backslash \hat{\mathscr{A}}_{m}}\left|\hat{\mathscr{L}}^{m} \chi_{\hat{\eta}}\right|\left(\hat{x}_{\hat{\eta}}\right) \quad \quad\left(=: \beta_{m}^{(3)}\right) \\
& +\sum_{i=1}^{\hat{N}(\Theta)} \sum_{\hat{\eta} \in \hat{\mathscr{Y}}_{m} \backslash \hat{\mathscr{A}}_{m}}\left|\left(\hat{\mathscr{P}}_{i} \hat{\mathscr{L}}^{m} \chi_{\hat{\eta}}\right)\left(\hat{x}_{\hat{\eta}}\right)\right| \quad\left(=: \beta_{m}^{(4)}\right) .
\end{aligned}
$$

Now, by the same reasoning as in (4.4),

and

$$
\beta_{m}^{(3)} \leqq \sum_{\substack{\hat{\eta} \in \hat{\mathscr{P}}_{m} \rightarrow \hat{\mathscr{A}}_{m} \\ \hat{\eta} \subseteq \hat{T}^{m} \hat{\eta}}} \sup _{\hat{\eta}}\left|\hat{g}_{m}\right| \leqq \text { const } \cdot \Theta^{m}
$$

$$
\begin{aligned}
\beta_{m}^{(4)} & \leqq \sum_{i=1}^{\hat{N}(\Theta)} \sum_{j=1}^{d_{i}} \sum_{\hat{\eta} \in \hat{\mathscr{X}}_{m} \backslash \hat{\mathscr{A}}_{m}}\left|\hat{\psi}_{i j}\left(\hat{\mathscr{L}}^{m} \chi_{\hat{\eta}}\right)\right|\left|\hat{\varphi}_{i j}\left(\hat{x}_{\hat{\eta}}\right)\right| \\
& \leqq \sum_{i=1}^{\hat{N}(\Theta)} \sum_{j=1}^{d_{i}} \sum_{\hat{\eta} \in \hat{\mathscr{X}}_{m} \hat{A}_{m}}\left\|\hat{\psi}_{i j}\right\| \cdot \operatorname{const} \cdot \Theta^{m} \cdot\left|\hat{\varphi}_{i j}\left(\hat{x}_{\hat{\eta}}\right)\right| \quad \text { by Corollary } 2.4 \\
& \leqq \text { const } \cdot \Theta^{m} \sum_{i=1}^{\hat{N}(\Theta)} \sum_{j=1}^{d_{i}} \sum_{\hat{C} \in \hat{\mathscr{D}}} \sup \left|\hat{\varphi}_{i j}\right| \leqq \operatorname{const} \cdot \Theta^{m} \sum_{i=1}^{\hat{N}(\Theta)} \sum_{j=1}^{d_{i}}\left\|\hat{\varphi}_{i j}\right\|_{B V} \\
& \leqq \text { const } \cdot \Theta^{m} .
\end{aligned}
$$

3. Let $0 \leqq k<m$. We first study

$$
\begin{aligned}
& \gamma_{m, k}^{(7)}=\sum_{\hat{\eta} \in \hat{\mathscr{Y}}_{m}} \operatorname{var}_{\hat{\eta}}\left(\hat{\mathscr{P}} \hat{Y}_{\hat{T}^{k}}\right)=\sum_{\eta^{\prime} \in \mathscr{X}_{m-k}} \sum_{\substack{\hat{\eta}^{\prime} \in \hat{\mathscr{Y}}_{m-k}^{2 m} \\
\pi \hat{\eta}^{\prime} \subseteq \eta^{\prime}}} \sum_{\substack{\hat{\eta} \in \hat{T}_{m} \\
\hat{T}^{\hat{\eta}} \hat{\eta}=\hat{\eta}^{\prime}}} \operatorname{var}_{\hat{\eta}}\left(\hat{\mathscr{P}} \hat{Y}_{\hat{\eta}^{\prime}}\right) \quad\left(=: \beta_{m}^{(5)}\right) \\
& +\sum_{\eta^{\prime} \in \mathscr{P}_{m-k}} \sum_{\hat{\eta}^{\prime} \in \hat{\mathscr{X}}_{m-k} \backslash \hat{\mathscr{X}}_{m-k}^{2 m}} \sum_{\substack{\hat{\eta} \in \hat{\eta}^{\prime} \subseteq \hat{\eta}^{\prime} \\
\hat{T}^{k} \hat{\eta}=\hat{\eta}^{\prime}}} \operatorname{var}_{\hat{\eta}}\left(\hat{\mathscr{P}} \hat{Y}_{\hat{\eta}^{\prime}}\right) \quad \quad\left(=: \beta_{m}^{(6)}\right) .
\end{aligned}
$$

The first term is bounded by:

$$
\beta_{m}^{(5)} \leqq \sum_{\eta^{\prime} \in \mathscr{\mathscr { P }}_{m-k}} \sum_{\substack{\hat{\eta}^{\prime} \in \hat{\mathscr{Y}}_{m-k}^{2 m} \\ \pi \hat{\eta}^{\prime} \subseteq \eta^{\prime}}} \operatorname{var}\left(\hat{\mathscr{P}} \hat{Y}_{\hat{\eta}^{\prime}}\right)
$$




$$
\begin{aligned}
& \leqq \sum_{\eta^{\prime} \in \mathscr{X}_{m-k}} \text { const } \cdot 2 m \cdot\|\hat{\mathscr{P}}\| \cdot v\left(\eta^{\prime}, m-k\right) \text { by (5.2) } \\
& \leqq \text { const } \cdot m \cdot \Theta^{m-k} .
\end{aligned}
$$

For the other term, we use the remark of part 1, and obtain:



$$
\begin{aligned}
& \leqq \sum_{i=1}^{\hat{N}(\Theta)} \sum_{j=1}^{d_{i}}\left\|\hat{\psi}_{i j}\right\| \sum_{\eta^{\prime} \in \mathscr{Z}_{m-k}} v\left(\eta^{\prime}, m-k\right) \sum_{\hat{\eta} \in \hat{\mathscr{Y}}_{m}} \operatorname{var}_{\hat{\eta}}\left(\hat{\varphi}_{i j}\right) \quad \text { by (5.2) } \\
& \leqq \sum_{i=1}^{\hat{N}(\Theta)} \sum_{j=1}^{d_{i}}\left\|\hat{\psi}_{i j}\right\| \cdot \operatorname{var}\left(\hat{\varphi}_{i j}\right) \cdot \text { const } \cdot \Theta^{m-k} \\
& \leqq \text { const } \cdot \Theta^{m-k} \text {. }
\end{aligned}
$$

We have thus proved that $\gamma_{m, k}^{(7)} \leqq$ const $\cdot \Theta^{m-k}$. Therefore:

$$
\begin{aligned}
\left|\gamma_{m}^{(3)}\right| & \leqq \sum_{\hat{\eta} \in \hat{\mathscr{X}}_{m}} \sum_{k=0}^{m-1}\left|\hat{g}_{k}\left(\hat{y}_{\hat{\eta}}\right)\right| \cdot \operatorname{var}_{\hat{\eta}}\left(\widehat{\mathscr{P}} \hat{Y}_{\hat{T}^{k}}\right) \\
& \leqq \sum_{k=0}^{m-1} \operatorname{const} \cdot \Theta^{k} \cdot \gamma_{m, k}^{(7)} \leqq \text { const } \cdot \Theta^{m}
\end{aligned}
$$

This shows the absolute convergence of $\gamma_{m}^{(3)}$.

4. The definition of $\hat{\mathscr{L}}$ yields:

$$
\begin{array}{rlr}
\left|\gamma_{m}^{(5)}\right| & =\left|\sum_{k=0}^{m-1} \sum_{\hat{\eta}^{\prime} \in \hat{\mathscr{X}}_{m-k}^{2 m}}\left(\hat{\mathscr{L}}^{k} \hat{\mathscr{P}} \hat{Y}_{\hat{\eta}^{\prime}}\right)\left(\hat{y}_{\hat{\eta}^{\prime}}\right)\right| \\
& \leqq \sum_{k=0}^{m-1} \operatorname{const} \cdot \Theta^{k} \cdot \sum_{\eta^{\prime} \in \mathscr{Z}_{m-k}} 2 \cdot m \cdot v\left(\eta^{\prime}, m-k\right) & \text { by Theorem } 1 \text { and }(5.2) \\
& \leqq \text { const } \cdot \Theta^{m} & \text { by }(5.3) .
\end{array}
$$

5. We use again the remark in 1 and obtain



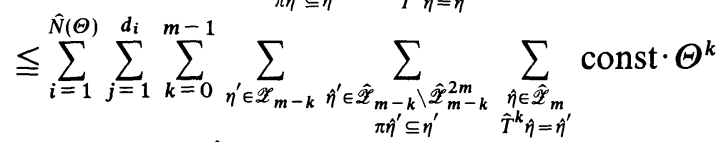

$$
\begin{aligned}
& \cdot\left|\hat{\varphi}_{i j}\left(\hat{y}_{\hat{\eta}}\right)\right| \cdot\left\|\hat{\psi}_{i j}\right\| \cdot v\left(\eta^{\prime}, m-k\right) \quad \text { by }(5.2) \\
& \leqq \text { const } \cdot \Theta^{k} \sum_{i=1}^{\hat{N}(\Theta)} \sum_{j=1}^{d_{i}} \sum_{k=0}^{m-1} \sum_{\eta^{\prime} \in \mathscr{Z}_{m-k}} v\left(\eta^{\prime}, m-k\right) \cdot\left\|\hat{\psi}_{i j}\right\| \cdot 2 \cdot\left\|\hat{\varphi}_{i j}\right\|_{\widehat{B} \boldsymbol{V}} \\
& \leqq \text { const } \cdot \Theta^{m} \\
& \text { by (5.3). }
\end{aligned}
$$


Acknowledgements. Both authors thank Jean-Pierre Eckmann for an invitation of G. Keller to Geneva which started this paper. The first author also wishes to express special thanks for constant encouragement. It is also a pleasure to thank David Ruelle for his notes on Haydn's proof and for suggesting study of this problem to the first author.

\section{References}

1. Baladi, V., Eckmann, J.-P., Ruelle, D.: Resonances for intermittent systems. Nonlinearity 2, 119-135 (1989)

2. Dunford, N., Schwartz, J. T.: Linear operators, part one. New York: Wiley 1957

3. Eckmann, J.-P.: Resonances in dynamical systems, Preprint. University of Geneva (1988)

4. Haydn, N. T. A.: Meromorphic extension of the zeta function for Axiom A flows. Preprint (1987)

5. Hofbauer, F.: On intrinsic ergodicity of piecewise monotonic transformations with positive entropy. Israel J. Math. 34, 213-237 (1979)

6. Hofbauer, F.: Periodic points for piecewise monotonic transformations. Ergod. Th. Dynam. Sys. 5, 237-256 (1985)

7. Hofbauer, F.: Piecewise invertible dynamical systems. Probab. Th. Rel. Fields 72, 359-386 (1986)

8. Hofbauer, F., Keller, G.: Ergodic properties of invariant measures for piecewise monotonic transformations. Math. Z. 180, 119-140 (1982)

9. Hofbauer, F., Keller, G.: Zeta-functions and transfer-operators for piecewise linear transformations. J. reine angew. Math. 352, 100-113 (1984)

10. Kato, T.: Perturbation theory for linear operators. Berlin, Heidelberg, New York: Springer 1976

11. Keller, G.: On the rate of convergence to equilibrium in one-dimensional systems. Commun. Math. Phys. 96, 181-193 (1984)

12. Keller, G.: Markov extensions, zeta-functions, and Fredholm theory for piecewise invertible dynamical systems. Preprint (1986), to appear in Trans. Am. Math. Soc.

13. Landau, E.: Darstellung und Begründung einiger neuerer Ergebnisse der Funktionentheorie. New York: Chelsea 1946

14. Milnor, J., Thurston, W.: On iterated maps of the interval. In: Dynamical systems (Lecture Notes in Mathematics vol. 1342) pp. 465-564. Berlin, Heidelberg, New York: Springer 1988

15. Mori, M.: On the Fredholm determinant of a piecewise linear transformation. Preprint, National Defense Academy of Japan (1987)

16. Pollicott, M.: Meromorphic extensions of generalised zeta functions. Invent. Math. 85, 147-164 (1986)

17. Preston, C.: What you need to know to knead, Preprint, University of Bielefeld (1988)

19. Ruelle, D.: Zeta functions for expanding maps aind Anosov flows. Invent. Math. 34, 231-242 (1976)

19. Ruelle, D.: Thermodynamic formalism. Reading MA: Addison-Wesley 1978

20. Ruelle, D.: One-dimensional Gibbs states and Axiom A diffeomorphisms. J. Diff. Geom. 25, 117-137 (1987)

21. Ruelle, D.: The thermodynamic formalism for expanding maps. Preprint (1989), Bowen lectures given at U.C. Berkeley in November 1988

22. Rychlik, M.: Bounded variation and invariant measures. Studia Math. LXXVI, 69-80 (1983)

23. Walters, P.: A variational principle for the pressure of continuous transformations. Am. J. Math. 97, 937-971 (1976)

\section{Communicated by J.-P. Eckmann}

Received November 4, 1988; in revised form July 7, 1989 
